\title{
A CONCURRENT ENGINEERING ENVIRONMENT IN THE CONSTRUCTION INDUSTRY
}

\author{
Suresh Babu M. ${ }^{1}$, Venkata Ramana V. ${ }^{2}$, Megharaj B.R. ${ }^{3}$ \\ ${ }^{1.2}$ Department of Mechanical Engineering, INTELL Engineering College, Anantapur, India \\ ${ }^{3}$ SKIM, Sri Krsihnadevaraya Universtiy, Anantapur, India. \\ Email: principaliis@rediff.com
}

\begin{abstract}
The construction industry has been moving towards concurrent engineering for many years. However, in practice, the degree of success achieved through the application of concurrent engineering varies considerably. Organizational factors, especially with respect to information management methods and techniques coupled with different levels of training and experience of personnel, place considerable limitations on the level of concurrency and collaborative work that can be achieved. In spite of these achievements, concurrent engineering issues, such as the management of the process, product documentation, and communication, are still being handled in a fragmented manner, as individual, mutually independent, or at best only partially interrelated systems. Interoperable environments have yet to be developed which can fully integrate into practice and increase the uptake of concurrent engineering.
\end{abstract}

Key words: Concurrent Engineering, Organizational factors, Level of Concurrency, interrelated systems

\section{INTRODUCTION}

Information technology has an enormous potential to improve both the organizational and the technological infrastructure of construction projects and thus to facilitate the effective application of concurrent engineering methodology. In the application of IT for concurrent engineering in building industry valuable steps have already been achieved in product model based integration, by STEP (ISO 10303-1), in process modeling, by the CALS initiative, in workflow developments and electronic document management by the WFMC as well as in several commercial and research based EDM systems.

In spite of these achievements, concurrent engineering issues, such as the management of the process, product documentation, and communication, are still being handled in a fragmented manner, as individual, mutually independent, or at best only partially interrelated systems. Interoperable environments have yet to be developed which can fully integrate into practice and increase the uptake of concurrent engineering.

To help tackle these issues a project called "Concurrent Engineering Environment in the Building and Engineering Structures Industry" has started. This project will contribute to the improvement of the organizational infrastructure for concurrent engineering by utilizing the opportunities offered by IT the primary objectives of the project are the development of an overall conceptual structure, as well as specific software tools, for concurrent engineering support. Some of the results are intended to be applied directly in the current environments utilized by end users. Other results will be developed and tested as prototypes leading to a more long term development approach.

\section{EXTENDING INTEGRATED ENVIRONMENTS FOR CONCURRENT ENGINEERING}

Before examining requirements to extend previously created integrated environment for concurrent engineering, it is worth considering what is meant by this term. From concurrent engineering research in other domains we have the following definitions:

In Dean and Unal (1992) concurrent engineering is defined as "getting the right people together at the right time to identify and resolve design problems. Concurrent engineering is designing for assembly, availability, cost, customer satisfaction, maintainability, manageability, manufacturability, operability, performance, quality, risk, safety, schedule, social acceptability, and all other attributes of the product".

It is widely accepted that concurrent engineering incorporates collaborative work, parallel product design advanced project management, consideration of the whole product life cycle, effective communication and information sharing across disciplines and phases of the product's development and adequate consideration of responsibilities Concurrent engineering also has major objectives to reduce the duration of an engineering project to increase the value of the product and at the same time reduce its cost and all these, partially contradictory objectives are pursued simultaneously and systematically.

In Winner et al. (1988) concurrent engineering is defined as "a systematic approach to the integrated, concurrent design of products and their related processes, including manufacturing and support. This approach is intended to cause the developers, from the outset, to consider all elements of the product life cycle from concept 
through disposal, including quality, cost, schedule and user requirements".

A range of progressive practitioners in the industry practices concurrent engineering today. However, these innovators receive little support from computerized tools for their efforts Though there has been a wide range of international funded projects developing frameworks for integration, concurrent engineering was seldom explicitly targeted The major concern of these projects was product data model development, and the associated data exchange and sharing which is a major task in itself. As these previous sponsored projects developed integrated design systems capable of exchanging data between ranges of design tools, it was realized that further support was necessary. It was seen that while exchange of data was useful, it was hard to tie in and utilize in actual projects if there was no association with the processes being managed in a project. This led to the incorporation of process models and processes management tools in a few of the later funded projects. However, while this made the integrated design systems more useable in a project, it still did not provide links to all information management aspects to be considered in a project. Missing from these systems, but of vital import in a project, is any consideration of documents and their prevalence in the communication of information in a real project.

Taking a consideration of documents brings in the legal aspects of a project including the implications of sending and receiving documents, and of signing them. Bringing together product, process and document models as well as the range of associated aspects provide benefits as listed below:

1. From an individual product it is possible to ascertain in which documents it is referred also in which processes and by which practitioners the product was designed and modified. The linkages also allow the history of design decisions to be tracked identifying who made which decisions, at which time and in which process.

2. From an individual document it is possible to ascertain the portion of the product model which is referenced by the document. In this way it is possible to identify who authorized a particular portion of the design and who was notified of the signed-off document. With a tracking of notifications and receipt and legal status codes for documents it is then possible to monitor and ascertain the legal status of all communications and presented information.

3. From a process view it is possible to identify the portion of the product which was modified model during the process and by which practitioners. It will also be possible to ascertain which documents were created or modified during the process.

The additional factors mentioned above extend the broad infrastructure of an integrated environment to better consider that which is seen in current projects. To support the requirements implicit in the descriptions of concurrent engineering provided above it is clear that an enabling framework must be able to support practitioners associated with a wide range of a building's life cycle. As well as defining process models which cover all these lifecycles, this argues for product data models which can represent information suitable for a wide range of practitioners at any one time.

\section{THE PROJECT "CONCURRENT ENGINEERING ENVIRONMENT" A STRUCTURE FOR CONCURRENT ENGINEERING}

The main goal of the project is the development of a conceptual structure and a prototype environment for support of concurrent engineering to be used by a wide range of institutions in the architectural engineering and construction domain. To achieve this goal the project builds upon the results and the acquired knowledge from many previous funded projects but extends the scope of research considerably to include several important concurrency enabling aspects with their mutual interrelationships. The focus of the project is especially on the holistic approach to design, on the management of the information flows in the multidisciplinary collaborative work processes, and on the legal aspects of electronic communication and information exchange.

The overall concept of a framework for concurrent engineering is being developed in acknowledgement of the following specific characteristics of the building industry:

1. Each major construction project is an example of a virtual corporation in business today. It is relatively common to find projects where the architect and major engineers are all from different countries and where the virtual product (i.e., the bid documents) of many hundreds of hours of highly skilled labor may exist as a complete package for the first time in the blue print shop. Even a relatively simple office building may involve companies from around the world. Although there may be examples of more complex projects being assembled by equally widespread teams, in no other industry would a team of such breadth be routinely dissolved after executing only a single copy of the product? 
2. Through the increased use of IT in the last few years, architecture and engineering have become typical information based professions, requiring because of the nature of their work highly decentralized information management solutions. During the design process large amounts of information are transferred electronically to and from, and processed by the architects, design consultants, contractors, owners and approval agencies. The coordination of that information and ensuring its understanding by the appropriate parties determines to a large degree the success or failure of a project. While perhaps less obvious to a person from the outside the role of a general contractor is very much the same.

Their success relies on coordinating the physical work of many subcontractors who may never have met or known each other prior to the project. The role of the project manager also as information manager is becoming more and more a determinant for the success of a project. However, communication and information exchange happen primarily between different organizations using applications and operating within different domains of $A / E / C$, and the scope of the exchanged data varies between nations, companies, projects project stages application systems and current view of the actors. A large variety of heterogeneous IT tools and a wide range of technologies are being used, and hence the needs of sending and receiving applications are not a priori predictable.

Because of this, project's main hypothesis is that any technology solution which is unduly centralized exclusive in terms of cost of computer expertise or in any way significantly restricting participation by the numerous small enterprises that make up a large part of the industry, could have unforeseen but not insignificant disadvantages and would not have good acceptance in practice.

There fore research focus is on the development of a fully open integration environment based on a clientserver architecture, using enabling Internet-technology and allowing the exploitation of various application tools each having its own information requirements and application specific data representations. The ability to mere transfer data between users or applications does not provide the full benefits which can be achievable from this technology. A wide range of issues must be supported in such an environment much greater than ever considered in previous projects. Key issues for a successful concurrent engineering approach being addressed in the project are:
1. Interoperability of distributed process, product, document and regulation models and data.

2. Information logistics and communication management

3. Inter-discipline conflict management

4. Monitoring and forecasting

5. Cost control.

6. Legal aspects related to the product data and the electronic documentation

Thus, several domain problems that were previously examined in isolation are being considered to provide a seamless prototype environment where a practitioner's full range of tasks will be inter-related allowing access to product information from documents, or documents for individual products and design tasks, and designers involved in particular decisions. Figure 1 shows a conceptual view of how these inter-related components can work together to provide the concurrent engineering environment.

All these aspects have to be modeled in all relevant subsystems of the concurrent engineering environment, and their constraints have to be taken into account adequately for all types of activities executed in a construction project. Legal aspects play a restrictive role to information management, and in fact in many ways reduce the possibilities to use available technology. However, if handled properly, they also provide an organizational rigor, which can help to reduce uncertainty and allocate correctly roles, responsibilities, decisions and co-ordination activities

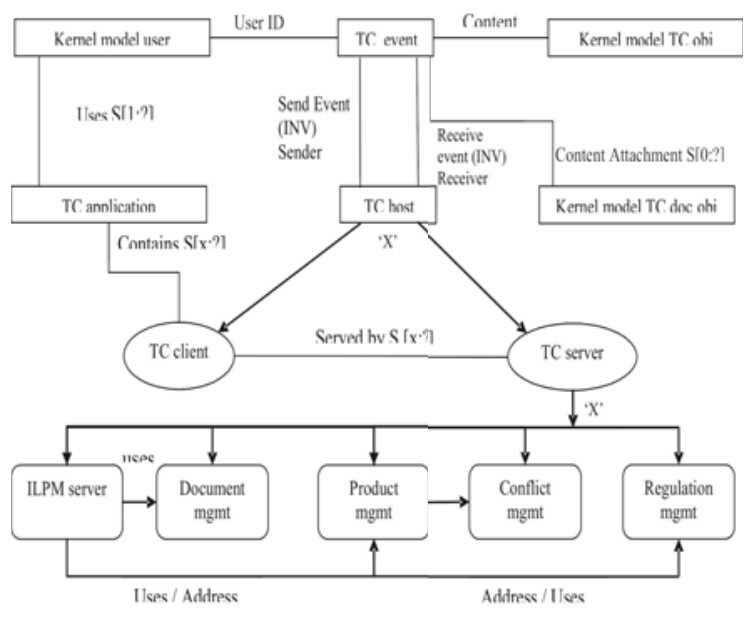

Fig. 1. The operational structure of concurrent engineering environment 


\section{A. The Modeling structure}

In order to achieve the interoperability of the heterogeneous modeling representations underlying the separate subsystems of the operational framework presented in Figure 1 develops a layered model architecture consisting of five hierarchically structured modeling abstractions. These are Meta Kernel, Neutral, Aspect and Application Models, as shown in Figure 2.

The Meta Model defines the logical schema of the basic modeling paradigm (extending EXPRESS) upon which all other models are built, i.e., the formal interpretation of all generic modeling concepts in this framework: Concept, Object, Class, Attribute, Object ID, Information, container, Access Right.

The Kernel Model defines the top level schema needed to link the different subsystems of concurrent engineering environment (process, product, document, conflict, regulations management). It provides unique and uniform specification of top level concepts for all information flows in the project process such as User, Client, Host, Communication Event, Project Object, Product Object etc.

These two layers and the Neutral Partial Models, binding the aspect and application models on the layers beneath, represent the Shared Project Model of concurrent engineering environment which will be 'visible' to all client applications and concurrent engineering services. Its objects will be accessible through common public methods that can be executed as remote procedure calls by any of the project subsystems and client applications.

The two lowest layers of the modeling framework represent the domain and application specific decomposition of the project data in a similar way to the approach taken in the international projects ATLAS and COMBI. However, in contrast to the shared Meta and Kernel models, these models need not be a priori harmonized. Their interoperability will be realized with the help of interface methods and not through specialization of the kernel objects. In this way, a federated structure can be established which does not impose the strict requirement for total logical consistency of all models.

With the introduction of this modeling framework the complexity of the interoperability problem is reduced to the intra-model-operability between the coherent sub models used in each individual subsystem and the inter-modeloperability.

1. Structural Engineering ---se1, se2, se3

2. Architect

3. HVAC

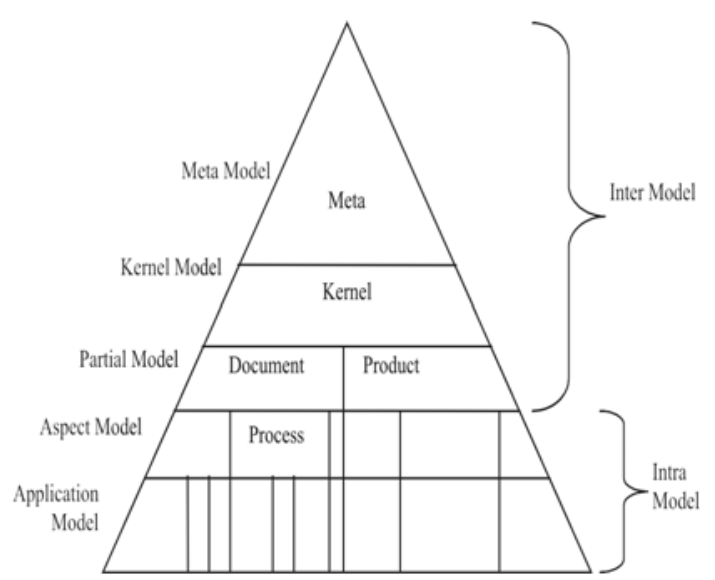

Fig. 2. The architecture model of Concurrent Engineering Environment

between the models of the different sub-systems which will be achieved with the help of the shared meta and kernel objects and a set of public interfaces

\section{B. The Process Model}

A system for control of the information flows for process supervision and guidance in design and construction and facility management must be integrated as a prime part of the targeted concurrent engineering environment. This system will be able to inter-relate the product model, project management and work flow information based on a common process model, which specifies the decomposition of the project goals into executable activities, and provides a logically coherent schema for the representation of high-level product, document and communication objects. However, since project information exists mainly in the form of product model data and as documents, the workflow methods and tools have to interact extensively with the product model and the document model management system. This type of information logistics demands a higher level of interoperability than just ensuring the data transformations and exchange between a set of partial product models, or the interrelations between the set of documents used in the project.

\section{The Product Model}

Though not yet very widely used in practice, product data technology is already available today. Product models at a high level of maturity have been developed in several research projects, e.g., in ATLAS COMBINE, COMBI. Through standardization efforts like the Building Construction Core Model 0 as well as through the IAI initiative (IAI 1996) product data technology is in fact on the threshold of wide scale commercial implementation. Because of this, it can be assumed in the near future 
product related information will be consistently represented in product data models developed according to the STEP methodology, such a model will provide the foundation for information sharing and exchange in construction projects. Therefore, a product model management system should be developed as an inherent part of the concurrent engineering environment. In concurrent engineering environment, the product model management system will build upon available achievements in product data technology, and will be able to fulfill the following objectives:

1. Provide an integrated set of layered product models to enable information sharing across discipline and over life cycle.

2. Include mechanisms for interoperability between the product models that need not necessarily be fully harmonized, by providing methods for both 'strong' and 'weak' integration

3. Include mechanisms for interoperability between product and document model data

4. Provide modeling support for application packages.

\section{The Document Model}

The product model infrastructure must be complemented by a document modeling and management system which fulfils also the legal requirements of project information management. While documents can be considered as views of the product model data, they need to cover more than because product information which is not contained in recognized legal documents is without legal validity in the context of inter-enterprise communication. Therefore a document management system is needed, which can capture such information management aspects as ownership, authentication, auditing, versioning, routing to actors etc.

A product and a document model have to co-exist and because of the requirements for their interRelationships are in fact highly influenced by each other. They need clearly defined interfaces built based on formal interoperability methods and recognized language specifications. However information exchange in a live project is to a certain extent always done through nonelectronic data. Such data have to be attached via the document model to the more tightly defined product model but cannot be automatically transformed into internal, computer-interpretable product information. They remain as external product information (as per STEP). Therefore, a mechanism for relating and attaching such data will be developed in concurrent engineering environment and will partly belong to the product model, which will be extended for such external attachments, and partly to the document model.

\section{E. Incorporation of LegalAspects}

Models and tools for the process, product and document aspects of project information are already developed to a high extent. Together with their integration into a logically consistent interoperable environment, another important, issue that is still missing is the consideration of the legal aspects of electronic communication and information management.

The current legal understanding in inter-enterprise information sharing is based on the following

\section{Propositions:}

The receiver has to receive the information in a reliable form and he has to be informed that he has to attach this information at a specific place following a specific procedure.

It is up to the sender to ensure that the receiver interprets the information exactly in the way the sender intends. This is achieved in current legal practice when the same, commonly accepted visual representation is used, e.g., text in an agreed language and graphical representations (technical drawings, schematic diagrams etc.) according to valid standards or best practice. In an automated system this can only be achieved electronically if the representation tools and the representation procedures (for the application of these tools) are connected in a 'legal' sense to the electronic data.

Other related aspects are legal security of the data, authorization on the data etc. responsibilities access rights and authorization control are being tackled with the help of specialized relationship objects associating the privileges of the actors in a project to any object, object group, process or document.

\section{F. The Regulations Model}

Building regulations and standards define the constraints to the building products and process. Ideally standards processing should actively restrict the set of possible solutions to a design problem to be subset conforming to the building regulations. This idealization implies that the full semantics of the building regulations can be captured by a computer program and used actively to check the solution set provided by the designer. In the last 30 years of related research, however, a general computable representation of the building codes has proven to be too complex.

Within concurrent engineering environment a scenario for the networked use of standard processing and conformance checking software consists of regulation 
servers, regulation clients, mapping servers and regulation agents. They are all located in a networked environment and use concurrent engineering logistics services to deliver several different views on the regulations, including hypertext and computable representations in the form of applets and code compliance checking services. The focus is on an open environment where different representations and tools may be used for different provisions, but conforming to the same minimal application interface. Clients to these services are both applications and engineers.

\section{G. Implementation of structure with an Information Logistics System}

Fig. 1 shows the operational structure of the concurrent engineering environment project. Some of its major components are discussed further below.

\section{Communication Management}

Concurrent engineering requires the control and monitoring of all information exchange activities, as far as they are not legally restricted. Therefore, the concurrent engineering environment should support all forms of electronic communication such as e-mail, file exchange or shared database access. Since most activities can be associated with (and will be defined in) the process model (for example, check-in and check-out of documents, RFIs, RFCs etc.), a tight integration of the communication services with the process model can greatly reduce the efforts for updating the project information.

In addition, the communication activities have also to be linked to an overall project database, where the data pertaining to possible senders and receivers should be appropriately specified and maintained.

Since communication management has to take into account the decentralized nature of $A / E / C$, an Internetenabled client-server approach covering distance and enterprise specific issues as well as hardware restrictions will be utilized in this project. The communication management system will make extensive use of the toplevel modeling objects of the framework (defined in the meta and the kernel and of a set of public functions for interfacing all other subsystems and visible to all actor and client applications.

\section{Conflict Management}

Enabling parallel work is one of the main benefits of concurrent engineering. However, this can inevitably lead to conflicts. Taking into consideration that the separate actors in a construction project usually work in their own discipline-specific environment, and decision making is mostly based on discipline specific aspects, data models and goals, another important requirement for an interoperable concurrent engineering environment is the development of conflict management methods to support interdisciplinary problem-solving issues, which cannot be localized within the limited scope of a single application system.

In this context, conflict management should be able to support the representation of high-level

Interdisciplinary constraints, and provide methods for conflict detection, co-ordination, and negotiation between the conflicting parties. The basic goal of conflict management should be to ensure consistency of the project data and hence to reduce re-work cycles and processing time. Thus, conflict management is an important enhancement to product data management and interoperability.

\section{DEMONSTRATION OF THE CONCURRENT ENGINEERING ENVIRONMENT SYSTEM}

An important part of the project is the validation and demonstration of the developed structure for concurrent engineering to a wide audience of specialists, so that a wide range of institutions in the architectural, engineering and construction domain can consider it.

A suitable real world case project and demonstration scenario has been selected in order to emphasize the key issues and the expected benefits. Setting up an appropriate demonstration scenario requires the following considerations.

1. A large scale engineering project is needed because several concurrent engineering aspects cannot be clearly expressed in relatively 'simple' projects, for example, a small office building.

2. The demonstration scenario should be able to show the holistic approach to design and construction which is typical for the concurrent engineering methodology by addressing important life cycle issues.

3. And covering interrelated aspects of design, construction and facility management.

4. The developed services, tools and application systems should be incorporated in a seamless process, because although such demonstration cannot cover all the complexity in a large-scale project, it has to be plausible and convincing, and inevitable simplifications must be reasonable.

5. The concurrent engineering environment a case project is on civil airport. The project is very large, involves a number of partners, both in design and 
construction works, and features the (typical for such projects) complexity of parallel activities, responsibilities, domain overlaps etc.

\section{CONCLUSIONS}

Concurrent engineering is not well supported with existing IT frameworks. The concurrent engineering environment project is tackling this problem through the development of a structure which ties together all information types and supports the full range of processes required for a concurrently engineered project. The project structure ties together product, process and document models enabling all project information to be managed and inter-related and also incorporates tools to manage the legality and authority of documents and processes, as well as functionality to manage conflicts between members in the project. Integrated functionality is also supported with design tools from a wide range of a building's lifecycle, as shown in the demonstration, along with code compliance checking and design tools. This complex system of tools is held together by an information logistics server which manages all flows of control and information in a project for practitioners from all firms involved in the project.

\section{REFERENCES}

[1] Amor, R. and Clift, M. (1996) Document Models and Concurrent Engineering in Turk, Z. (ed) 'Construction on the Information Highway', Proc. CIB-W78 Workshop, Bled'96.

[2] Augenbroe G. (ed) (1995) COMBINE Final Report, EU/CEC Joule Programme, Project JOU2-CT920196, TU Delft.

[3] bohms M. and Storer, Architecture, methodology and Tools for computer-integrated Large Scale engineering, in Proc. JSPE-IFIP WG 5.3 Workshop, DIISM'93, Tokyo.

[4] Dean, E.B. and Unal, R. (1992) Elements of Designing for Cost, in Proc. AIAA 1992 aerospace Design AConference, AIAA-92-1057, Irvinc,CA, USA

[5] ESPRIT IV-20587 ToCEE (1995) EU ESPRIT IV Project 20587 ToCEE - Project programme EU/CEC, ProgDirectorate Generale III, Brussels
[6] IAI (1996) End User Guide to Industry Foundation Classes, International Alliance for Interoperability, Version 1.0.

[7] ISO 10303-1 (1994) Product Data Representation and Exchange - Part 1: Overview and Fundamental Principles, ISO TC 184/SC4, Geneva, 17p.

[8] ISO 10303-41, 45, 103, -106, -225, -230, -231 (199296): Product Data Representation and Exchange Parts 41-45, 103, 106, 225, 230, 231, ISO TC 184/SC4, NIST.

[9] Jablonski, S. (1995) Workflow-ManagementSysteme: Motivation, Modellierung, Archite ktur (in German), Informatik Spektrum 18(1).

[10] Katranuschkov, P., Scherer, R.J., Clift, M. and Amor, R. (1996) EU ESPRIT IV Project 20587 ToCEE Deliverable J.1 'Migration Perspectives', Public Report, EU/CEC, Directorate Generale III, Brussels.

[11] Scherer, R.J. and Sparacello, H.-M. (eds) (1996) COMBI Final Report, EU/CEC ESPRIT III Project No. 6609, TU Dresden.

[12] Watson, A. and Crowley, A. (1995) CIMsteel Integration Standards, in R.J.Scherer (ed) "Product and Process Modelling in the Building Industry", Proc. of ECPPM'94, Dresden, Balkema, pp 491-494.

[13] Winner, R.I., Pennell, J.P., Bertrand, H.E. and Slusarezuk, M.M.G. (1988) The Role of Concurrent Engineering in Weapon Systems Acquisition, Institute for Defense Analysis, IDAReport R-338

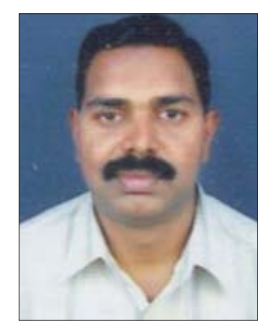

Mr. M.Suresh Babu is the Principal of Intel Institute of Science, Anantapur. He obtained his Master's in Computer Application from Osmania University and M.Phil Degree in Computer Science from Bharathiar University. His field of research is "Computer Science on Inductive Data Mining". He has organized several workshops and training Programmes in the field of Computer Science. He has published 20 Technical Papers in various Conferences and Journals. 